\title{
MODELOS DE RENDIMIENTO PARA Eucalyptus globulus (Labill) EN BASE A VARIABLES AMBIENTALES
}

\author{
Letelier, Luis ${ }^{1}$, Higuera, Cristian ${ }^{2}$ y Real, Pedro ${ }^{3}$.
}

\section{RESUMEN}

En este estudio se evaluó el comportamiento de modelos tradicionales de altura dominante y área basal incorporando variables ambientales para plantaciones de Eucalyptus globulus en la zona sur de Chile.

Para la incorporación de las variables ambientales, se determinó la cantidad de radiación solar neta acumulada en cada período de crecimiento en cada una de las parcelas consideradas. Esta variable ingresa al modelo en remplazo de la edad del rodal y en su cálculo se considera la incorporación de modificadores que dependen de las temperaturas y precipitaciones en conjunto con las características del suelo y propias de la especie. El cálculo de los modificadores se basa en los submodelos ambientales del modelo de proceso 3PG.

Se compararon los modelos generados con los modelos empíricos utilizados en la zona de estudio por el simulador EUCASIM, comprobándose para altura dominante una mejora en las proyecciones realizadas con el nuevo modelo mientras que en el caso del área basal los resultados fueron similares en ambos tipos de modelos.

La inclusión de variables ambientales en los modelos empíricos y el uso de submodelos desarrollados en el contexto de modelos de procesos, como técnica de hibridación, pueden ser de utilidad ya que, además de mejorar las estimaciones, el modelo híbrido se hace sensible a perturbaciones climáticas extremas como períodos de sequía o heladas.

Los resultados obtenidos son promisorios para el desarrollo de este tipo de modelos y reducen la incertidumbre de las predicciones en escenarios de cambio climático y sectores de transición de zonas de crecimiento.

Se considera necesario profundizar en este tipo de modelos, ya que pueden convertirse en un apoyo en las decisiones que se tomen ante condiciones de cambio ambiental o en el análisis de nuevas áreas de plantaciones.

Palabras clave: Eucalyptus globulus, Crecimiento, Modelo Híbrido, Recursos Acumulados

\footnotetext{
${ }^{1}$ Modelo Nacional de Simulación, Facultad de Ciencias Forestales, Universidad de Concepción, Chile lletelier@udec.cl

${ }^{2}$ Modelo Nacional de Simulación, Facultad de Ciencias Forestales, Universidad de Concepción, Chile chiguera@udec.cl

${ }^{3}$ Facultad de Ciencias Forestales, Universidad de Concepción, Chile preal@udec.cl
} 


\section{ABSTRACT}

In this study, traditional models for dominant height and basal area using the environmental variable effect were evaluated for Eucalyptus globulus in south-central Chile.

To incorporate environmental variables in the models, the amount of net solar radiation for each measurement in each plot was determined. This variable was added in the model by replacing the stand age and its value consider growth modifiers which depend on the temperature, precipitation, soil and species properties. These modifiers were calculated based on environmental submodels from 3PG process model.

The generated models were compared with the empirical models used in the study area by the EUCASIM simulator, proving an improved with the new model in dominant height, while in basal area with both empirical and hybrid models found similar results.

The inclusion of environmental variables in the empirical models and use of submodels developed in de process model context as a hybridizing method maybe useful, because improves the estimated value, because with this type of variables the model is sensitive to extreme climatic disturbances like drought or frost period.

The results of this study are promissory to development of such models and they reduce the uncertainty of estimates in climate change scenarios and transition section of the growth zone.

This type of models should be better studied because it can be converted into a tool for decision support considering scenarios of change of environmental conditions or the analysis of new plantation areas.

Keywords: Eucalyptus globulus, Growth, Hybrid Model, Accumulated Resources 


\section{INTRODUCCIÓN}

Desde los inicios del Modelo Nacional de Simulación se han empleado modelos empíricos para realizar proyecciones de crecimiento o rendimiento, los que han sido mejorados con la inclusión de nuevos ensayos y mediciones. Dichos modelos han sido ajustados para cada una de las zonas de crecimiento definidas en el simulador en base a condiciones ambientales homogéneas. Sin embargo, según algunos autores los modelos empíricos resultan ser poco sensibles a variaciones climáticas (Mason et al, 2008). Otro de los inconvenientes en este tipo de estratificación es la diferencia en productividad en sectores límites entre zonas de crecimiento.

En general en los modelos empíricos se utiliza la edad como variable de entrada para representar el crecimiento de un rodal (Mason et al., 2011), sin embargo, la edad representa una acumulación constante de tiempo y el bosque, al depender de condiciones del ambiente, no crece en forma constante a lo largo de un período. Otra opción para obtener proyecciones más detalladas pueden ser los modelos basados en procesos fisiológicos. Existe un interés creciente en el uso de los modelos de proceso, de los que destaca el modelo 3PG, no precisamente por ser un modelo superior a otros, sino por ser un modelo relativamente simple y de libre disposición (Sands, 2004). Este modelo cuenta con cinco submodelos que son: producción de biomasa, fijación de biomasa (entre follaje, raíces y fuste), mortalidad, balance de agua en el suelo y módulo para convertir biomasa fustal en variables de interés (Sands, 2004). El modelo necesita información climática como variables de entrada y trabaja en forma mensual o anual (Landsberg y Waring, 1997).

Una alternativa para mejorar las proyecciones y hacer que los modelos sean sensibles a variaciones climáticas extremas es utilizar la metodología de hibridación propuesta por Mason et al. (2011), en la que se reemplaza la variable edad por la cantidad de recursos acumulados, la cual está definida por la cantidad de radiación neta que recibe un rodal, corregido por modificadores de crecimiento que dependen de las temperaturas y precipitación en conjunto con las características del suelo y propias de la especie. Los modificadores recogen las variaciones climáticas y limitan el crecimiento en períodos en los que las características ambientales no son las adecuadas para el desarrollo óptimo del rodal. El cálculo de los modificadores se basa en los submodelos ambientales del modelo de proceso 3PG. Con esta metodología se puede dar solución al problema de diferencias de productividad en los límites de las zonas de crecimiento. En el presente trabajo se evaluó la metodología de hibridación aplicada a los modelos de altura dominante y área basal, comparando los resultados con un modelo empírico actualmente en uso para la especie.

\section{MATERIAL Y METODO}

\section{Descripción de los Datos}

Se utilizaron los datos de ensayos y parcelas permanentes instalados por el Modelo Nacional de Simulación de Eucalipto, que se encuentran distribuidos en casi la totalidad de la zona sur de Chile.

Debido a la gran extensión del territorio considerado, el área fue dividida en zonas de crecimiento sobre las cuales el Modelo Nacional de Simulación ha trabajado en modelos empíricos que actualmente se encuentran disponibles en el simulador EUCASIM versión 2011. En total se contó con 489 parcelas y el resumen de la información se muestra en el Cuadro $\mathrm{N}^{\circ} 1$.

A esta información dasométrica se asoció información climática, obtenida de la Dirección General de Aguas (DGA), como radiación solar, temperaturas y precipitaciones, que está en forma mensual calculada como el promedio de los diez años comprendidos en el período 1998-2008. Además, se agregaron datos de las condiciones del suelo tal como capacidad de agua aprovechable y tipo de suelo provenientes de las series de suelo del CIREN. 
Cuadro $\mathrm{N}^{\circ} 1$

ESTADÍSTICOS DESCRIPTIVOS DE LOS DATOS UTILIZADOS EN EL AJUSTE DE LOS MODELOS

\begin{tabular}{|c|c|c|c|c|c|}
\hline $\begin{array}{l}\text { Pares } \\
\text { de } \\
\text { Datos }\end{array}$ & Variable & Mínimo & Máximo & Promedio & $\begin{array}{c}\text { Desviación } \\
\text { Estándar }\end{array}$ \\
\hline \multirow{5}{*}{3345} & $\begin{array}{l}\text { Edad } \\
\text { (años) }\end{array}$ & 3,01 & 19,00 & 7,51 & 3,40 \\
\hline & $\begin{array}{l}\text { Altura Dominante }(\mathrm{H} 100) \\
(\mathrm{m})\end{array}$ & 3,50 & 46,38 & 20,93 & 7,67 \\
\hline & Número de árboles (NHA) & 157,50 & 2990,00 & 1422,56 & 431,77 \\
\hline & $\begin{array}{l}\text { Área Basal (GHA) } \\
\left(\mathrm{m}^{2} / \mathrm{ha}\right)\end{array}$ & 0,60 & 78,46 & 23,97 & 11,68 \\
\hline & $\begin{array}{l}\text { Volumen total sin corteza (VHA) } \\
\left(\mathrm{m}^{3} / \mathrm{ha}\right)\end{array}$ & 0,20 & 852,60 & 188,33 & 145,35 \\
\hline
\end{tabular}

Cada uno de los pares de datos fue validado en su consistencia respecto a cada una de las variables de la base de datos que son necesarias para los modelos.

Esta información, junto a los datos topográficos provenientes de un Modelo de Elevación Digital (DEM por sus siglas en inglés), es la base para la interpolación a las coordenadas geográficas de cada parcela, obteniéndose así la información climatológica necesaria para describir el punto a proyectar. Para esto se utilizó el método ThinPlateSmoothingSpline (TPSS) implementado en ANUSPLIN V4.36 (Hutchinson y Gessler, 1994), interpolando las variables de interés a cada una de las parcelas del proyecto.

\section{Especificación de los Modelos}

Los modelos empíricos actualmente implementados en el simulador EUCASIM fueron hibridados al reemplazar la edad por el valor de radiación acumulada al momento de la predicción, corregida por modificadores que dependen de las temperaturas y precipitaciones en conjunto con las características propias del suelo. Los modificadores se calcularon mediante el uso de los submodelos ambientales del modelo de proceso 3PG, la expresión que representa la acumulación de recursos se muestra en la Fórmula 1.

$$
R_{T}=\sum_{t=1}^{T} \operatorname{rad}_{t} *\left(f_{\text {temp }} * \min \left\{f_{v p d} f_{s w}\right\}\right)
$$

Donde: $\quad R_{T}=$ Recursos acumulados al momento $\mathrm{T}$.

$\mathrm{rad}_{t}=$ Radiación en el mes t.

$f_{\text {temp }}=$ Modificador de temperatura.

$f_{v p d}=$ Modificador por déficit de presión de vapor.

$f_{s w}=$ Modificador por agua aprovechable en el suelo.

Los modificadores de temperatura, déficit de presión de vapor y agua del suelo, se obtienen mediante la aplicación de las Fórmulas 2, 3 y 4, respectivamente.

$$
f_{\text {temp }}=\left(\frac{T a_{i}-T \min }{T \circ p t-T \min }\right) *\left(\frac{T \max -T a_{i}}{T \max -T o p t}\right)^{\frac{\left.(T \max -T o p)^{2}\right)}{(T o p t-T \min \eta)}}
$$




$$
\begin{aligned}
& f_{\text {DPd }}=e^{\left(-0.05 * V P D_{i}\right)} \\
& f_{\text {SV }}=\frac{1}{1+\left(\frac{1-\left(\frac{N w_{i}}{N_{\max }}\right)}{\varphi_{\theta}}\right)^{n_{\theta}}}
\end{aligned}
$$

$$
\begin{aligned}
\text { Donde: } V P D_{i} & =\text { Déficit de presión de vapor en el mes } \mathrm{i} . \\
T a_{i} & =\text { Temperatura media mensual para el mes i. } \\
T \text { min } & =\text { Temperatura mínima de crecimiento para la especie. } \\
T \text { max } & =\text { Temperatura máxima de crecimiento para la especie. } \\
T o p t & =\text { Temperatura óptima de crecimiento para la especie. } \\
s w_{i} & =\text { Agua en el suelo disponible para el mes } \mathrm{i} . \\
s w_{\max } & =\text { Máxima cantidad de agua disponible en el suelo. } \\
c_{\ominus} \text { y } n_{\ominus} & =\text { Constantes que toman diferentes valores según el tipo } \\
& \text { de suelo. }
\end{aligned}
$$

En el modelo de altura dominante se adaptó la metodología del índice de sitio, para ello se determinó un índice de productividad (ISR) que corresponde a la altura promedio de los 100 árboles más grandes cuando el rodal ha acumulado cierta cantidad de radiación. A este nivel de radiación se le puede llamar Radiación Clave $\left(R_{c}\right)$ quedando la siguiente expresión de cálculo para este índice:

$$
I S R=a_{0} *\left(1-\left(1-\left(\frac{H 100}{a_{0}}\right)^{a_{1}}\right)^{\left(\frac{R_{c}}{R}\right)}\right)^{\left(\frac{1}{a_{1}}\right)}
$$

Una vez calculado este índice de productividad, se puede calcular la altura dominante para cualquier nivel de radiación utilizando el siguiente modelo:

$$
H 100=a_{0} *\left(1-\left(1-\left(\frac{I S R}{a_{0}}\right)^{a_{1}}\right)^{\left(\frac{R}{R_{c}}\right)}\right)^{\left(\frac{1}{a_{1}}\right)}
$$

En el modelo de área basal, se debe determinar el nivel de recurso acumulado para un momento inicial y un momento final, para luego estimar el área basal en el momento final de la siguiente forma:

$$
\left.\left.G_{f}=G_{i}^{\left(\frac{a_{i}}{a_{f}}\right)^{b_{2}}} * e^{\left[\left(\frac{b_{0}}{b_{2}}+\frac{b_{1}}{b_{2}} *\left(H 100 f_{f} * R_{f}\right)\right.\right.}\right) \cdot\left(1-\left(\frac{a_{i}}{R_{f}}\right)^{b_{2}}\right)\right]
$$

Donde: $\quad H 100=$ Altura promedio de los 100 árboles más grandes del rodal.

$R \quad=$ Radiación acumulada .

$R_{c} \quad=$ Radiación clave .

$I S R$ = Índice de productividad por radiación, corresponde a la altura promedio de los 100 árboles dominantes cuando el rodal ha alcanzado la radiación clave.

$G_{i}, G_{f}=$ Área basal en los momentos inicial y final respectivamente.

$a_{0}, a_{1}, b_{0}, b_{1}$ y $b_{2}$ corresponden a los coeficientes de cada modelo. 


\section{Validación de los Modelos}

El proceso de validación consistió en comparar la capacidad predictiva de los modelos híbridos con la de los modelos empíricos implementados en EUCASIM. Se realizaron distintas simulaciones considerando una medición específica como momento inicial y las mediciones siguientes como momentos finales; el proceso fue repetido para cada medición con el fin de cuantificar el error y sesgo asociados al largo de las proyecciones en la predicción de altura dominante y área basal.

Para determinar el error y sesgo asociado a las simulaciones se utilizaron los estadísticos Error Cuadrático Medio (ECM) y Diferencia Agregada (DIFA); estas medidas se calcularon por clase de edad y por cantidad de períodos de proyección. La expresión de las medidas antes mencionadas es la siguiente:

$$
\begin{aligned}
& E C M=\frac{\sum\left(O_{i}-E_{i}\right)^{2}}{n} \\
& D I F A=\frac{\sum\left(O_{i}-E_{i}\right)}{n}
\end{aligned}
$$

Donde: $\quad O_{\mathrm{i}}=$ Valor observado.

$E_{\mathrm{i}}=$ Valor estimado.

$n=$ Número de observaciones.

\section{RESULTADOS}

\section{Modelo de Altura Dominante}

Se encontró un menor error, para todas las clases de edad, con el modelo híbrido, obteniendo un máximo de $6,01 \%$ a los 10 años. Mientras que con el modelo empírico, el máximo se produce a la misma edad, alcanzando el 7,33\%. En el caso del sesgo, ambos modelos se muestran insesgados, no sobrepasando en ningún caso el $\pm 1,5 \%$. El detalle de error y sesgo según clase de edad puede apreciarse en el Cuadro $\mathrm{N}^{\circ} 2$.

Cuadro $\mathrm{N}^{\circ} 2$

ERROR CUADRÁTICO MEDIO (ECM) Y DIFERENCIA AGREGADA (DIFA) SEGÚN CLASE DE EDAD PARA ALTURA DOMINANTE

\begin{tabular}{|c|c|c|c|c|}
\hline \multirow{2}{*}{$\begin{array}{c}\text { Clase de Edad } \\
\text { (años) }\end{array}$} & \multicolumn{2}{|c|}{$\begin{array}{c}\text { ECM } \\
\text { (\%) }\end{array}$} & \multicolumn{2}{c|}{$\begin{array}{c}\text { DIFA } \\
\text { (\%) }\end{array}$} \\
\cline { 2 - 5 } & Empírico & Híbrido & Empírico & Híbrido \\
\hline 5 & 5,11 & 5,09 & $-0,19$ & 0,47 \\
\hline 10 & 7,33 & 6,01 & $-0,55$ & $-0,71$ \\
\hline 15 & 7,04 & 5,93 & 1,37 & $-0,05$ \\
\hline 20 & 7,10 & 5,82 & 0,60 & $-0,28$ \\
\hline
\end{tabular}


Para el largo del período de proyección se observa claramente una mejora en el error del modelo híbrido sobre el empírico.

Ambos modelos tienen bajo error con períodos de proyección cortos, pero se incrementan a medida que aumenta la cantidad de períodos.

Al llegar a los 16 períodos, el modelo empírico alcanza un 15,86\%, mientras que el híbrido solo tiene un $10,56 \%$ de error.

En cuanto al sesgo, la tendencia es similar, pero al superar los 12 períodos de proyección, con el modelo empírico se encuentran sesgos superiores al 7\%, alcanzando el 11,03\% en los 16 períodos, mientras que con el híbrido para igual largo de proyección se alcanza solo el $5,86 \%$.

Los resultados para el largo del período de proyección se encuentran en el Cuadro $N^{\circ} 3$.

Cuadro $\mathbf{N}^{\circ} 3$

ERROR CUADRÁTICO MEDIO (ECM) Y DIFERENCIA AGREGADA (DIFA) SEGÚN PERÍODOS DE PROYECCIÓN PARA ALTURA DOMINANTE

\begin{tabular}{|c|c|c|c|c|}
\hline \multirow{2}{*}{$\begin{array}{c}\text { Períodos de } \\
\text { Proyección }\end{array}$} & \multicolumn{2}{|c|}{$\begin{array}{c}c \\
\text { (\%) }\end{array}$} & \multicolumn{2}{c|}{ (\%) } \\
\cline { 2 - 5 } & Empírico & Híbrido & Empírico & Híbrido \\
\hline 2 & 4,23 & 3,94 & $-0,20$ & $-0,22$ \\
\hline 4 & 7,38 & 6,21 & $-0,45$ & $-0,93$ \\
\hline 6 & 8,27 & 6,94 & 0,84 & $-0,76$ \\
\hline 8 & 9,17 & 7,55 & 2,13 & $-0,24$ \\
\hline 10 & 10,34 & 7,99 & 3,11 & 0,44 \\
\hline 12 & 11,18 & 8,70 & 4,03 & 1,99 \\
\hline 14 & 14,03 & 10,05 & 7,58 & 4,24 \\
\hline 16 & 15,86 & 10,52 & 11,03 & 5,86 \\
\hline
\end{tabular}

En las Figuras $\mathrm{N}^{\circ} 1$ y $\mathrm{N}^{\circ} 2$ se puede observar una mejor distribución de los residuales con el modelo híbrido en el cual, la mayor parte de los residuos se centra dentro de los \pm 5 metros, mientras que para el modelo empírico estos se sitúan entre los \pm 10 metros.

Esto se refleja en que los valores estimados por el modelo híbrido se ajustan mejor a la curva 1:1 que la del modelo empírico. 

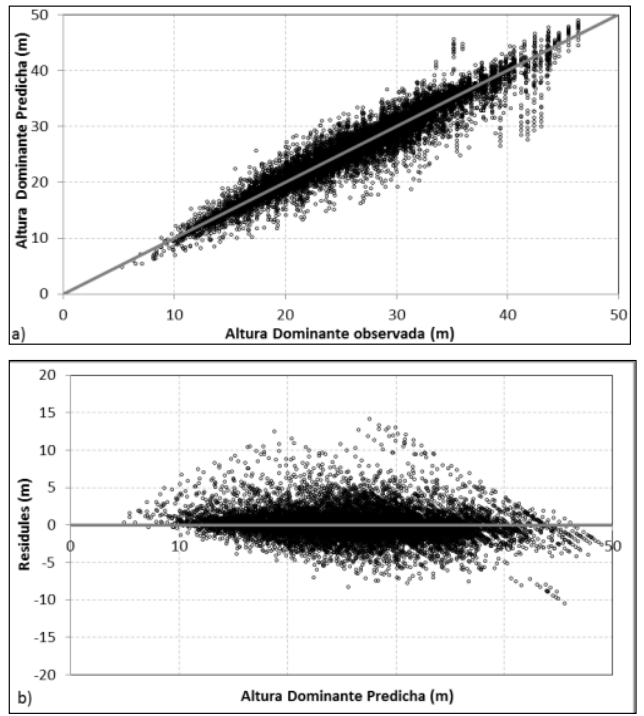

Figura $\mathbf{N}^{\circ} 1$

GRÁFICOS DE VALIDACIÓN DE ALTURA DOMINANTE PARA EL MODELO EMPÍRICO A) VALORES PREDICHOS VERSUS OBSERVADOS Y B) RESIDUALES VERSUS VALORES PREDICHOS
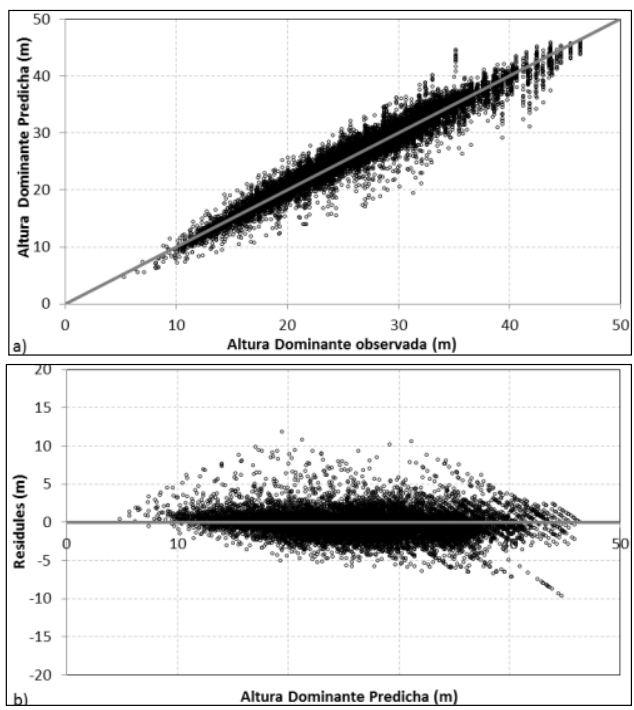

Figura $\mathbf{N}^{\circ} 2$

GRÁFICOS DE VALIDACIÓN DE ALTURA DOMINANTE PARA EL MODELO HÍBRIDO A) VALORES PREDICHOS VERSUS OBSERVADOS Y B) RESIDUALES VERSUS VALORES PREDICHOS 


\section{Área Basal}

En área basal no se aprecian diferencias entre los resultados obtenidos con ambos tipos de modelos, el error es similar para cada clase de edad, el que se sitúa alrededor del $8 \%$, alcanzando un máximo en la clase de 20 años con un 11\%. La mayor diferencia se encuentra a los 5 años donde el modelo empírico presenta un error de 5,91\% y el híbrido de 7,06\%. Para el sesgo, en ambos casos, solo supera al $2 \%$ en la clase de 20 años, para el resto de las clases apenas se alcanza el $\pm 1 \%$, estos resultados se presentan en el Cuadro $\mathrm{N} 4$.

Cuadro $\mathrm{N}^{\circ} 4$

ERROR CUADRÁTICO MEDIO (ECM) Y DIFERENCIA AGREGADA (DIFA) SEGÚN CLASE DE EDAD PARA ÁREA BASAL

\begin{tabular}{|c|c|c|c|c|}
\hline \multirow{2}{*}{$\begin{array}{c}\text { Clase de Edad } \\
\text { (años) }\end{array}$} & \multicolumn{2}{|c|}{$\begin{array}{c}\text { ECM } \\
\text { (\%) }\end{array}$} & \multicolumn{2}{c|}{$\begin{array}{c}\text { DIFA } \\
\text { (\%) }\end{array}$} \\
\cline { 2 - 5 } & Empírico & Híbrido & Empírico & Híbrido \\
\hline 5 & 5,91 & 7,06 & 0,08 & 1,52 \\
\hline 10 & 8,06 & 8,13 & $-1,00$ & $-0,15$ \\
\hline 15 & 8,83 & 8,93 & $-0,36$ & $-0,20$ \\
\hline 20 & 11,11 & 11,28 & 2,89 & 3,91 \\
\hline
\end{tabular}

Al analizar el largo del período de proyección, la similitud de los modelos se hace más evidente. Para cada largo de período, el error alcanzado por el híbrido no supera al empírico en más de un $1 \%$. En el caso del sesgo, hasta los 10 períodos de proyección ambos modelos se muestran completamente insesgados, mientras que para períodos de mayor longitud se supera el $5 \%$ de sesgo, alcanzando el 9,93\% con el modelo híbrido y el $8,75 \%$ con el modelo empírico en los 14 períodos de proyección. En el Cuadro $N^{\circ} 5$ se muestran los resultados descritos.

\section{Cuadro $\mathrm{N}^{\circ} 5$ \\ ERROR CUADRÁTICO MEDIO (ECM) Y DIFERENCIA AGREGADA (DIFA) SEGÚN PERÍODOS DE PROYECCIÓN PARA ÁREA BASAL}

\begin{tabular}{|c|c|c|c|c|}
\hline \multirow{2}{*}{$\begin{array}{c}\text { Períodos de } \\
\text { Proyección }\end{array}$} & \multicolumn{2}{|c|}{$\begin{array}{c}c \mid \\
\text { ECM } \\
\text { (\%) }\end{array}$} & \multicolumn{2}{c|}{} \\
\cline { 2 - 5 } & Empírico & Híbrido & Empírico & Híbrido \\
\hline 2 & 4,21 & 4,29 & $-0,29$ & 0,11 \\
\hline 4 & 8,36 & 8,47 & $-0,96$ & $-0,07$ \\
\hline 6 & 10,72 & 11,01 & $-0,69$ & $-0,03$ \\
\hline 8 & 12,62 & 12,93 & 0,30 & 0,90 \\
\hline 10 & 14,56 & 14,63 & 2,56 & 3,55 \\
\hline 12 & 17,30 & 17,15 & 5,78 & 7,07 \\
\hline 14 & 18,83 & 18,48 & 8,75 & 9,93 \\
\hline 16 & 15,52 & 15,99 & 5,67 & 7,27 \\
\hline
\end{tabular}

En las Figuras $\mathrm{N}^{\circ} 3$ y $\mathrm{N}^{\circ} 4$ se puede observar una dispersión similar entre los modelos al graficar los valores estimados con los observados. Además, la forma de la distribución de los residuales de ambos modelos presenta un mismo patrón, sin embargo, con el modelo híbrido los residuos se concentran un poco más cercanos al eje. Las curvas 1:1 de ambos modelos no muestran mayores diferencias. 

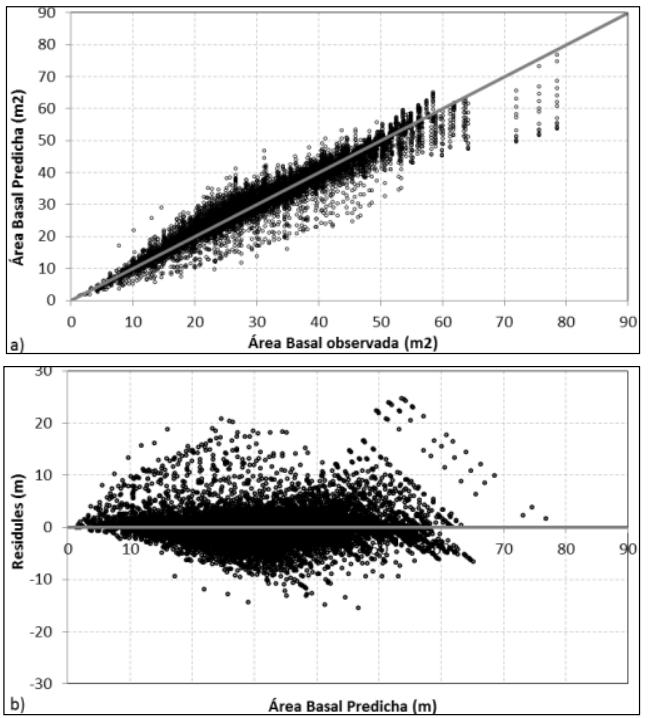

Figura $\mathrm{N}^{\circ} 3$

GRÁFICOS DE VALIDACIÓN DE ÁREA BASAL PARA EL MODELO EMPÍRICO

A) VALORES PREDICHOS VERSUS OBSERVADOS Y B) RESIDUALES VERSUS VALORES PREDICHOS
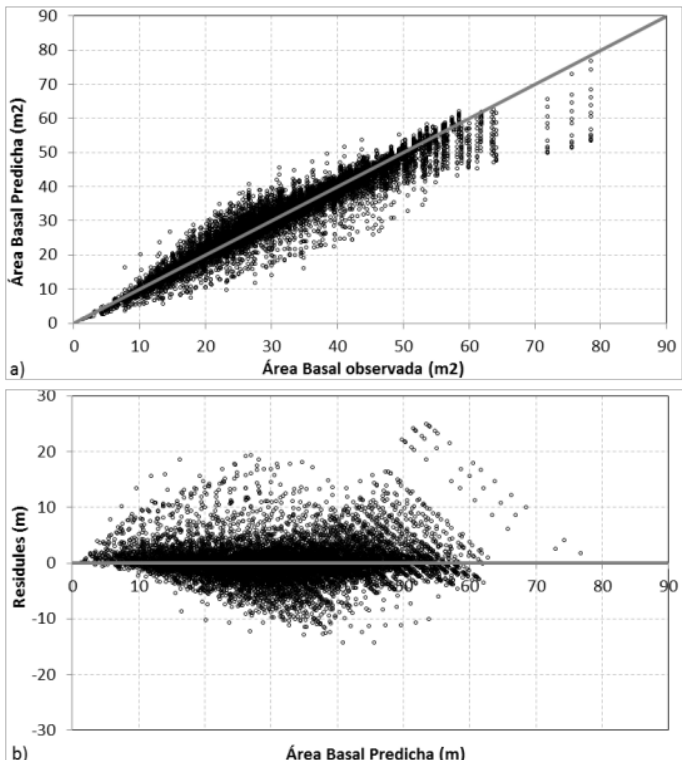

Área Basal Predicha (m)

Figura $\mathrm{N}^{\circ} 4$

GRÁFICOS DE VALIDACIÓN DE ÁREA BASAL PARA EL MODELO HÍBRIDO

A) VALORES PREDICHOS VERSUS OBSERVADOS Y B) RESIDUALES VERSUS VALORES PREDICHOS 


\section{CONCLUSIONES}

Se evaluó una opción de hibridación para altura dominante y área basal, la que fue contrastada con los respectivos modelos empíricos que se encuentra disponibles en el simulador EUCASIM 2011. Del análisis realizado se puede concluir que la metodología de hibridación empleada resulta ser factible de implementar, pero la obtención y posterior manipulación de la información para generar los niveles de recursos acumulados puede no ser una etapa sencilla. En el análisis realizado se utilizaron los submodelos ambientales de modelo 3PG, esto hizo necesaria una calibración previa del 3PG para la obtención de los modificadores crecimiento. Como desafío futuro sería interesante evaluar la inclusión de modelos que reemplacen total o parcialmente algunos aspectos que dependen de la calibración del 3PG, como por ejemplo el índice de área foliar.

Los modelos, en general, tuvieron un buen comportamiento. En el caso de altura dominante se logró mejorar las proyecciones con el modelo híbrido, aunque el empírico también mostró buenos resultados. En las proyecciones de área basal, ambos modelos presentaron resultados similares.

En este trabajo solo se probó la hibridación con un modelo, el que está implementado en el simulador EUCASIM, lo que hace recomendable que este análisis pueda extenderse en etapas posteriores a la evaluación de nuevos modelos. El trabajar con modelos que incorporen variables ambientales debiera eliminar el efecto de las zonas de crecimiento, ya que en cada parcela o rodal a proyectar se incorporan variables que representan las condiciones ambientales propias de su localización geográfica.

Sin duda es necesario profundizar en este tipo de modelos, pues pueden convertirse en una herramienta poderosa a la hora de evaluar el desarrollo de los rodales ante condiciones climáticas cambiantes, en el análisis de nuevas áreas de plantaciones, o en la proyección de sectores ubicados en áreas límites de zonas de crecimiento.

\section{REFERENCIAS}

Hutchinson, M.F. and Gessler, P. E., 1994. Splines - more than just a smooth interpolator. Geoderma 62: 45-67.

Landsberg, J. J. and Waring, R. H., 1997. A generalised model of forest productivity using simplified concepts of radiation-use efficiency, carbon balance and partitioning. ForestEcology and Management, 95, 209-228.

Mason, E. G., Woollons, R. C. and Manley, B. R., 2008. Carbon accounting: Forest growth rates and changing climates. Ministry of Agriculture and Forestry, 47 p. New Zeland.

Mason, E. G., Menthol, R. and Cochrane, H., 2011. Hybrid mensurational and physiological modelling of growth and yield of Pinus radiate D. Don using potentially useable radiation sums. Forestry, 84(2).99-108.

Sands, P., 2004. Adaptation of 3PG to novel species: guidelines for data collection and parameter asignment. Tecnical report 141, CRC for Sustainable Production Forestry, Hobart, Australia. 
